\title{
CRISPR-Cas13 as a tool for RNA interference
}

Zahir Ali ${ }^{1}$, Ahmed Mahas ${ }^{1}$, and Magdy Mahfouz ${ }^{1, *}$

${ }^{1}$ Laboratory for Genome Engineering, Division of Biological Sciences, 4700 King Abdullah University of Science and Technology, Thuwal 23955-6900, Saudi Arabia.

*Corresponding author: Magdy M. Mahfouz (magdy.mahfouz@kaust.edu.sa)

Key words: CRISPR-Cas13, RNA manipulation, viral interference, transcriptome regulation 


\begin{abstract}
:
Almost all biological processes involve RNA, making it crucial to develop tools for manipulation of the transcriptome. The bacterial CRISPR-Cas13 system was recently rewired to facilitate RNA manipulation in eukaryotes, including plants. Here, we discuss the opportunities and limitations of using CRISPR-Cas13 in plants for various types of RNA manipulation.
\end{abstract}

Besides being a major factor in protein production (mRNA, rRNA, tRNA), RNA can regulate gene expression (non-coding RNAs) [1]. The broad importance of RNA prompted the development of innovative approaches for targeted RNA manipulation, such as RNA interference, to regulate RNA transcript abundance, control viral infections, alter epigenetic states, and increase transcriptome plasticity to enhance plant survival in unfavorable environments [1]. However, additional RNAtargeting tools are needed to examine RNA-related mechanisms and their applications.

\title{
The CRISPR-Cas system as a tool for RNA manipulation
}

CRISPR-Cas systems serve as powerful tools for DNA editing, using a CRISPR RNA (crRNA) to provide targeting specificity. Recently, the newly identified class II type VI CRISPR-Cas13 systems were found to be amenable for use in RNA manipulation in eukaryotic cells [2, 3]. Cas13 proteins contain two Higher Eukaryotes and Prokaryotes Nucleotide-binding RNase domains that mediate target RNA cleavage [4]. Cas13 can process its own pre-crRNAs, allowing individual short single crRNAs to be customized to target RNA in vitro or to provide Escherichia coli with programmable immunity against ssRNA-lytic MS2 phage (Box 1) [3, 4].

\section{Application of CRISPR-Cas13 in eukaryotes}

CRISPR-Cas13 can target specific endogenous RNAs and viral RNAs in mammalian cells and plants. Recent studies identified Cas13 variants with high RNA targeting efficiency and specificity, which they used for targeted knockdown of endogenous transcripts in mammalian cells and plant protoplasts [5]. In addition, Cox et al. expanded the RNA targeting ability of Cas13 to direct ADAR2 deaminase for RNA-base (adenosine to inosine) modifications in human cells to recover functional proteins and halt disease progression [6, 7]. Aman et al. used CRISPRLshCas13a to confer modest resistance against an RNA virus in plants, providing evidence that this system could be used for biotechnological and agricultural applications $[5,8]$. 


\section{The current state of CRISPR-Cas13 technology}

These studies point to the promise of developing a transformative RNA targeting and manipulation tool. However, this use of CRISPR-Cas13 remains in its infancy; further investigation will be needed to take this technology into the next stage. Cox et al. tested several CRISPR-Cas13 systems and found that PspCas13b is the most efficient Cas13 protein. This study was carried out only in mammalian cells; investigation of different CRISPR-Cas13 systems and other proteins in plants might identify a Cas 13 variant with more robust, specific activity for RNA manipulation in planta. In addition, extensive biochemical and structural analyses are needed to understand the mode of action of Cas13 in different plant species, which could result in the generation of a Cas13 variant optimized for use in plants. Thus, there is a great need to further characterize and optimize RNA targeting in plants to make it highly effective, efficient and precise.

\section{Potential uses for CRISPR-Cas13 in plants}

Specific targeting of RNA

Compared to small RNAs and RNA interference, which are difficult in design and are limited by high off-target potential, CRISPR-Cas13 can be used to manipulate only the target RNA, with few or no off-target effects in eukaryotes, and multiple crRNAs can be used to eradicate a particular mRNA transcript (Figure 1a).

\section{Cas13 processing of pre-crRNA}

The innate ability of Cas13 to process pre-crRNA into individual crRNAs [4] can facilitate simultaneous targeting of several mRNAs involved in one or multiple pathways to help fieldgrown plants cope with many simultaneous stresses or pathogens. Such gene silencing would be of great value in plant organelles in which other RNA manipulation systems do not work or are not available. Cas13 can target a single virus at multiple sites or multiple RNA viruses simultaneously (Figure 1b). Importantly, even some of the newly evolved viral variants can still be targeted, as Cas13 tolerates single-base mismatches and cleaves targets outside of its recognition sequence [4].

Cas13 as a diagnostic tool 
Plants in the field often face mixed pathogenic infections, and identifying individual pathogens is challenging. Cas13a can be used to detect particular RNAs [7], which could be useful for identifying the precise cause of pathogenicity, particularly at early stages of infection in the field (Figure 1c).

\section{Cas 13 can limit pathogenicity}

Plants employ programmed cell death (PCD) as a defense mechanism. Successful targeting by Cas13a in E. coli results in collateral nonspecific RNA degradation, pointing to the potential applications of PCD [3, 7]. A CRISPR-Cas13 variant with robust nonspecific RNA degradation activity can target single or multiple pathogens by triggering PCD in plant cells, thereby restricting infection (Figure 1d).

\section{dCas13 as an RNA targeting platform}

Targeted, noninvasive RNA modification and viral interference hold great promise, especially using an inducible CRISPR-Cas13 system to alter RNAs involved in biotic or abiotic stress or silencing an RNA virus. Like dCas9, dCas13 retains its specific RNA-binding programmability [6]. The binding of dCas13-crRNA complexes can hinder viral replication and direct specific modifications to different RNAs. For example, dCas13 can be fused to a deaminase that can convert adenosine to inosine (Inosine reads as Guanosine) to recover functional proteins, skip the virus replication machinery (Figure 1e), or increase plant transcriptome plasticity to improve stress tolerance through customized alternative splicing (Figure 1f). Also, epigenetic or fluorescent tags can be fused to dCas13 to direct specific epitranscriptomic changes or to visualize mRNA trafficking to specific cellular organelles (Figure 1g).

\section{Potential limitations of using CRISPR-Cas13 in plants}

CRISRP-Cas13 systems have numerous advantages for agriculture owing to their simplicity, robustness, and ability for multiplex targeting. However, three possible obstacles might hinder the usefulness of CRISPR-Cas13. (i) Collateral RNA degradation. Even though promiscuous RNA degradation by Cas 13 was not observed in the three studies in human cells and plants described above, initial studies performed in vitro and in bacterial cells showed that after binding to its target, Cas13 nonspecifically degrades RNA [4]. The biochemistry of Cas13 in eukaryotic cells must be 
better understood before CRISPR-Cas13s can be used in crop plants. Possible solutions to such obstacles include inactivating the catalytic domain responsible for promiscuous degradation and engineering dCas13 fused to a ribonuclease domain (such as the PINc domain) to generate a dCas13-PIN platform capable of precise targeting and controlled RNA degradation. (ii) Possible off-target activity of CRISPR/Cas13. Like Cas9 [9], an enhanced variant of Cas13 with better specificity could be engineered, or different modalities of crRNAs could be assayed to enhance specific targeting. (iii) Can viral targeting using Cas13 accelerate the emergence of new viruses? A better understanding of the extent to which CRISPR-Cas13 can cope with recombinant viral escapes is required, and careful planning is needed to avoid the potential risks of such recombinant viruses in breeding programs.

\section{Concluding Remarks}

Recent findings suggest that, like CRISPR-Cas9 for DNA manipulation and engineering viral resistance [10-12], CRISPR-Cas13 holds great promise as a robust, precise, scalable RNAtargeting platform for RNA manipulations and RNA virus targeting in plants [5, 8]. We hope that in response to the growing need for such a tool, rigorous studies will establish this system as the gold standard for RNA manipulation in plants. Such a method would provide a variety of opportunities for addressing major agricultural and food security challenges.

\section{Acknowledgements}

We wish to thank the Office of Sponsored Research (OSR), King Abdullah University of Science and Technology (KAUST) for their support (Award No. OSR-2015-CRG4-2647).

\section{References:}


1. Liu, X., et al., Long Non-coding RNAs and Their Biological Roles in Plants. Genomics, Proteomics \& Bioinformatics, 2015. 13(3): p. 137-147.

2. Mahas, A., C. Neal Stewart Jr, and M.M. Mahfouz, Harnessing CRISPR/Cas systems for programmable transcriptional and post-transcriptional regulation. Biotechnology Advances.

3. Abudayyeh, O.O., et al., C2c2 is a single-component programmable RNA-guided RNAtargeting CRISPR effector. Science, 2016. 353(6299).

4. East-Seletsky, A., et al., Two distinct RNase activities of CRISPR-C2c2 enable guide-RNA processing and RNA detection. Nature, 2016. 538(7624): p. 270-273.

5. Abudayyeh, O.O., et al., RNA targeting with CRISPR-Cas13. Nature, 2017. 550(7675): p. 280-284.

6. Cox, D.B.T., et al., RNA editing with CRISPR-Cas13. Science, 2017.

7. Gootenberg, J.S., et al., Nucleic acid detection with CRISPR-Cas13a/C2c2. Science, 2017. 356(6336): p. 438-442.

8. Aman, R., et al., RNA virus interference via CRISPR/Cas13a system in plants. Genome Biology, 2018. 19(1): p. 1.

9. $\mathrm{Fu}, \mathrm{Y}$., et al., Improving CRISPR-Cas nuclease specificity using truncated guide RNAs. Nature biotechnology, 2014. 32(3): p. 279-284.

10. Ali, Z., et al., Efficient Virus-Mediated Genome Editing in Plants Using the CRISPR/Cas9 System. Molecular Plant. 8(8): p. 1288-1291.

11. Ali, Z., et al., CRISPR/Cas9-mediated viral interference in plants. Genome Biol, 2015. 16: p. 238.

12. Ali, Z., et al., CRISPR/Cas9-Mediated Immunity to Geminiviruses: Differential Interference and Evasion. Scientific Reports, 2016. 6: p. 26912.

\section{Box 1. The bacterial CRISPR-Cas13 system against RNA phages}

Prokaryotic cells have evolved innate defense mechanisms to protect themselves against nucleic acids derived from bacteriophages and other foreign genetic elements. The CRISPR-Cas 13 system functions as an "adaptive" immune system in bacteria and archaea to fend off invading RNA elements, such as RNA viruses, by recognizing the invading RNAs and mediating their subsequent degradation [3,4]. On the first encounter with RNA phages, CRISPR-Cas13 systems acquire a short (28-30 nt) spacer sequence from the phage genome and incorporate it in their CRISPR array through an unknown mechanism. Bacterial acquired immunity occurs via three steps (see Figure I): (i) expression, where the CRISPR array, along with Cas13, are transcribed and Cas 13 protein is produced; (ii) processing, where pre-crRNA is processed by Cas13 to form individual, short crRNAs (28-30 nt spacers and 28-36 nt Cas13 binding scaffold); and (iii) targeting or viral interference, where the invading phage RNA is cleaved by the crRNA-Cas13 complex. Upon binding to its target, Cas13 promiscuously degrades cellular RNA, leading to PCD [3, 4]. 
Figure 1. Potential applications of CRISPR-Cas13 in plant biotechnology. (A) Targeted RNA cleavage. Specific RNA molecules can be targeted by single or multiple crRNAs generated from long pre-crRNA transcript via Cas13. Such multiplexed targeting can provide additive effect on gene knockdown. (B) Resistance against RNA viruses. CRISPR-Cas13 can be harnessed for single [8] or simultaneous targeting of multiple RNA viruses, which would be highly valuable for crops in areas with mixed endemic viruses. (C) Specific mRNA or viral species detection. The collateral activity of CRISPR-Cas13 can be used to detect a specific RNA or a particular pathogen in a pool of mixed samples through utilization of the target RNA binding-dependent nonspecific degradation of quench-labeled RNA reporters and the subsequent release of florescence signal [7]. (D) Engineering programmed cell death (PCD). PCD (or hypersensitive response) can be mimicked for a broad range of pathogens via binding of Cas13 to pathogenic target and the collateral degradation of cellular RNA. (E) RNA base editing. dCas13-deaminase fusions [6] can be used to recover functional proteins, alter RNA viral genomes or to skip viral replication machinery. (F) pre-mRNA programed alternative-splicing. dCas13 fused to deaminases or splicing factors can be used for customized alternative-splicing to allow plants to tolerate a particular stress condition. (G) Endogenous mRNA imaging and localization. dCas13 fused to florescent protein can be utilized to detect the localization of transcript/s inside plant cell [2]. Also, dCas13 fused to nuclear, mitochondrial, chloroplast, or cytoplasmic localization signals can transport mRNA to the desired cellular organelles. 